\title{
Rotationally resolved electronic spectroscopy of 5-methoxyindole
}

\author{
Christian Brand, ${ }^{1}$ Olivia Oeltermann, ${ }^{1}$ David Pratt, ${ }^{2}$ Rainer Weinkauf, ${ }^{1}$ W. Leo Meerts, ${ }^{3}$ \\ Wim van der Zande, ${ }^{3}$ Karl Kleinermanns, ${ }^{1}$ and Michael Schmitt ${ }^{1, a)}$ \\ ${ }_{1}^{1}$ Institut für Physikalische Chemie I, Heinrich-Heine-Universität, D-40225 Düsseldorf, Germany \\ ${ }^{2}$ Department of Chemistry, University of Pittsburgh, Pittsburgh, Pennsylvania 15260, USA \\ ${ }^{3}$ Institute for Molecules and Materials, Radboud University, Heyendaalseweg 135, NL-6525 AJ Nijmegen, \\ The Netherlands
}

(Received 4 May 2010; accepted 10 June 2010; published online 9 July 2010)

\begin{abstract}
Rotationally resolved electronic spectra of the vibrationless origin and of eight vibronic bands of 5 -methoxyindole (5MOI) have been measured and analyzed using an evolutionary strategy approach. The experimental results are compared to the results of ab initio calculations. All vibronic bands can be explained by absorption of a single conformer, which unambiguously has been shown to be the anti-conformer from its rotational constants and excitation energy. For both anti- and syn-conformers, a ${ }^{1} L_{a} /{ }^{1} L_{b}$ gap larger than $4000 \mathrm{~cm}^{-1}$ is calculated, making the vibronic coupling between both states very small, thereby explaining why the spectrum of 5MOI is very different from that of the parent molecule, indole. (0) 2010 American Institute of Physics. [doi:10.1063/1.3459128]
\end{abstract}

\section{INTRODUCTION}

Indole, the parent molecule of 5-methoxyindole (5MOI), has been the subject of a large number of experimental ${ }^{1-7}$ and theoretical ${ }^{8-12}$ studies. This large interest mainly arises from the fact that indole is the chromophore of the aromatic amino acid tryptophan, whose fluorescence is an important probe of the local surroundings in proteins. ${ }^{13,14}$ Indole and its derivatives have two close-lying $\pi \pi^{*}$ states, which exhibit very different excited state dipole moments. They are called ${ }^{1} L_{a}$ and ${ }^{1} L_{b}$ in the nomenclature of Platt, ${ }^{15}$ adapted to indole by Weber. ${ }^{16}$ The ${ }^{1} L_{b}$ state has a small dipole moment and is lower in energy in apolar surroundings, while the ${ }^{1} L_{a}$ state, on the other hand, has a comparatively large dipole moment, leading to an inversion of the electronic state ordering in polar solvents. To gain a greater insight into the interplay between these strongly overlapping electronic states, the chromophore indole has been studied thoroughly. The main focus of this work has been the location of the ${ }^{1} L_{a}$ origin, about which there has been much controversy for several decades. The difficulty in the analysis arises from the fact that these states are vibronically coupled. Therefore, a transition exhibiting the electronic nature of the ${ }^{1} L_{a}$ state could either be the origin or a vibration of this state, or alternatively a vibration of the ${ }^{1} L_{b}$ manifold vibronically coupled to the ${ }^{1} L_{a}$ state.

Recently, we have examined the vibronic coupling in indole thoroughly by a combination of theory and rotationally resolved electronic spectroscopy in the gas phase. ${ }^{17,18}$ In these studies we showed that up to $1000 \mathrm{~cm}^{-1}$ above the origin, all bands in the spectrum of indole belong to the ${ }^{1} L_{b}$ state but are vibronically coupled to the ${ }^{1} L_{a}$ state to varying degrees.

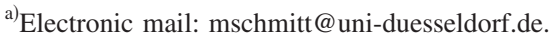

The energy separation between the lowest electronically excited singlet states can be changed by introducing substituents to indole; methyl and methoxy groups (depending on their position) stabilize one of the electronic states, leading in some cases to a change of the energetic ordering in the gas phase. $^{2}$ The largest energy separation of these derivatives is found for 5MOI. Owing to a large redshift of its ${ }^{1} L_{b}$ state, the ${ }^{1} L_{a} /{ }^{1} L_{b}$ gap increases to $3088 \mathrm{~cm}^{-1}$, as shown by Martinaud and Kadiri ${ }^{19}$ by comparing solvent induced shifts of the UVabsorption in di-n-propyl ether and polyethylene films. Methoxy substituents additionally have an influence on the angle $\theta_{a b}$ between the ${ }^{1} L_{a}$ and ${ }^{1} L_{b}$ transition dipole moment vectors, as shown by Eftink et al. ${ }^{2}$ This value is nearly $90^{\circ}$ for the unsubstituted indole. ${ }^{20}$ Albinsson and Nordén ${ }^{3}$ determined the angle $\theta_{a b}$ between the transition dipole moments of the ${ }^{1} L_{a}$ and the ${ }^{1} L_{a}$ states to be $85^{\circ}$.

Resonance enhanced two-photon ionization (R2PI) spectra of jet-cooled 5MOI have been measured by Hager et al. ${ }^{21}$ up to $1200 \mathrm{~cm}^{-1}$ above the electronic origin. Excited state lifetimes were determined for various vibronic bands. In a study of Huang et al., ${ }^{22}$ R2PI spectra of 5MOI and of 1:1 and 1:2 adducts with water, methanol, and triethylamine were presented. Florio et al. ${ }^{23}$ showed by UV-UV holeburning spectroscopy that all bands in the laser induced fluorescence (LIF) spectrum of 5MOI arise from a single ground state level, which they assigned to the anti-methoxy conformer (see Fig. 1).

Here, we present the results of an analysis of the electronic origin as well as of several vibronic bands of 5MOI with rotational resolution. The spectra were analyzed using an evolutionary strategy (ES) approach and the resulting molecular parameters were compared to the results of quantum chemical calculations. Our objective is to contrast the characters of its electronic states with those of the parent molecule, indole. 

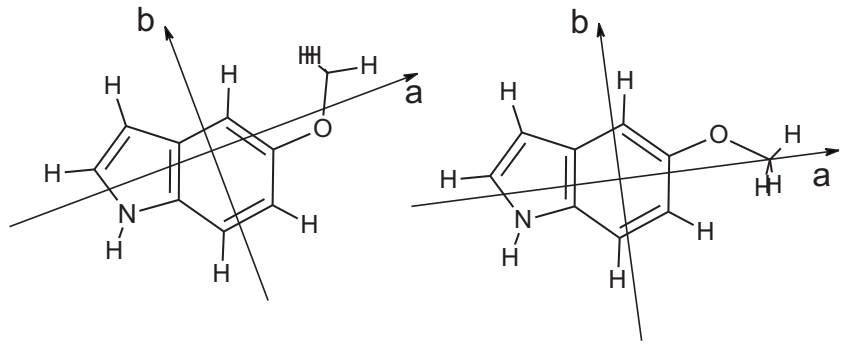

FIG. 1. Structures and inertial axes of anti- (left) and syn- (right) 5MOI.

\section{TECHNIQUES}

\section{A. Experimental procedures}

The experimental setup for the R2PI experiment is described in detail elsewhere. ${ }^{24}$ In brief, the sample molecules were heated to $110^{\circ} \mathrm{C}$ to form a suitable partial pressure in a pulsed valve (General Valve, Iota One) operated at $10 \mathrm{~Hz}$ with 4 bar $\mathrm{Ar}$ as a carrier gas. The pulsed supersonic beam was skimmed after $30 \mathrm{~mm}$ and ionized about $200 \mathrm{~mm}$ downstream of the valve in a second vacuum chamber. Ions were accelerated in a two-stage ion source and mass detected in a linear time-of-flight mass spectrometer. The spectra were taken by recording the dependence of the gated ion signal on the laser wavelength. Excitation light was provided by a frequency-doubled pulsed neodymium-doped yttrium aluminum garnet (Nd:YAG) (Innolas Spitfire 600) pumped dye laser (Lumonics Hyperdye 500) with a 7 ns pulse width.

5-methoxyindole ( $\geq 99 \%)$ was purchased from Fluka and used without further purification. The experimental setup for the rotationally resolved laser induced fluorescence is described in detail elsewhere. ${ }^{25}$ In brief, the laser system consists of a single frequency ring dye laser (Sirah Matisse DS) operated with rhodamine $6 \mathrm{G}$, pumped with $6 \mathrm{~W}$ of the 532 $\mathrm{nm}$ line of a frequency-doubled cw Nd:YAG laser (Spectra). The dye laser output was coupled into an external folded ring cavity (Spectra Physics Wavetrain) for the second harmonic generation. The resulting output power was constant at about $25 \mathrm{~mW}$ during each experiment. The molecular beam was formed by coexpanding 5MOI, heated to $170{ }^{\circ} \mathrm{C}$, and 800 mbar of argon through a $80 \mu \mathrm{m}$ nozzle into the vacuum chamber. The molecular beam machine consists of three differentially pumped vacuum chambers that are linearly connected by skimmers ( 1 and $3 \mathrm{~mm}$, respectively) in order to reduce the Doppler width. The resulting resolution is 18 $\mathrm{MHz}$ (full width at half maximum) in this setup. In the third chamber, $360 \mathrm{~mm}$ downstream of the nozzle, the molecular beam crosses the laser beam at a right angle. The imaging optics setup consists of a concave mirror and two planoconvex lenses to focus the resulting fluorescence onto a photomultiplier tube, which is mounted perpendicularly to the plane defined by the laser and molecular beam. The signal output was then discriminated and digitized by a photon counter and transmitted to a PC for data recording and processing. The relative frequency was determined with a quasiconfocal Fabry-Pérot interferometer. The absolute frequency was obtained by comparing the recorded spectrum to the tabulated lines in the iodine absorption spectrum. ${ }^{26}$

\section{B. Computational methods}

\section{Quantum chemical calculations}

Structure optimizations were performed employing the valence triple-zeta basis set with polarization functions $(\mathrm{d}, \mathrm{p})$ from the TURBOMOLE library. ${ }^{27,28}$ The equilibrium geometries of the electronic ground and the lowest excited singlet states were optimized using the approximate coupled cluster singles and doubles model (CC2) employing the resolutionof-the-identity approximation. ${ }^{29-31}$

Singlet state energies and wave functions were calculated using the combined density functional theory/ multireference configuration interaction (DFT/MRCI) method developed by Grimme and Waletzke. ${ }^{32}$ Configuration state functions (CSFs) in the MRCI expansion were constructed from Kohn-Sham (KS) orbitals, optimized for the dominant closed shell determinant of the electronic ground state employing the BH-LYP ${ }^{33,34}$ functional. All 56 valence electrons were correlated in the MRCI runs and the eigenvalues and eigenvectors of four singlet states were determined. The initial set of reference configuration state functions was generated automatically in a complete active space type procedure (including all single and double excitations from the five highest occupied molecular orbitals in the KS determinant to the five lowest virtual orbitals) and was then iteratively improved. The MRCI expansion was kept moderate by extensive configuration selection. The selection of the most important CSFs is based on an energy gap criterion, as described in Ref. 32. Only those configurations were taken into account that have an energy below a certain cutoff energy. The energy of a given configuration was estimated from orbital energies within the selection procedure. The cutoff energy was given by the energy of the highest desired root as calculated for the reference space plus a cutoff parameter $\delta E_{\text {sel }}=1.0 E_{\mathrm{h}}$. The latter choice has been shown to yield nearly converged results. ${ }^{32}$

\section{Fits of the rovibronic spectra using evolutionary algorithms}

The search algorithm employed to fit the rotationally resolved electronic spectra was an evolutionary strategy adapting normal mutations via a covariance matrix adaptation (CMA-ES) mechanism. This algorithm was developed by Ostenmeier and Hansen ${ }^{35,36}$ and is designed especially for optimization on rugged search landscapes that are additionally complicated due to noise, local minima, and/or sharp bends. It belongs, like the other search algorithms we use, to the group of global optimizers that were inspired by natural evolution. The schemes of these optimizers are quite similar to each other and will be approximately described in the following paragraph.

Trial solutions, which contain all the information needed to describe a spectrum (individuals), are generated and evaluated according to their correlation with the experimental spectrum (evaluation). Based on this result, the following generation is constructed (selection and reproduction). The actual method of reproduction depends on the type of algorithm; in genetic algorithms, chosen solutions of the former generation exchange parameters to create new solutions. In 
evolutionary strategies, on the other hand, new solutions are generated as a random distribution around a single point in search space. For the CMA-ES, this point (the geometrical mean $\mathbf{m}$ ) is computed from a weighted sum of selected individuals from the foregoing generation. The weighting procedure favors good solutions and should move the mean $\mathbf{m}$ toward the expected minimum of the potential surface. Afterward, this new generation is again evaluated. This circle of generating and evaluating individuals goes on until the process converges to the solution of the problem.

The main difference between the CMA-ES algorithm and earlier algorithms we have used is the employment of the covariance matrix. The covariance matrix is expected to shape the distribution of the generated individuals in such a way that it adapts to the multidimensional function space. For example, if the potential surface resembles a valley with respect to a certain parameter, the covariance matrix shapes the distribution to mainly follow the valley. Estimated from scratch during the first generation, the matrix is updated and consequently improved as generations accumulate. The objective of the matrix adaptation is to appropriate the inverse Hessian, which is assumed to be the optimal covariance matrix.

As the covariance matrix determines the shape of the distribution, the actual step-size is evaluated independently. The criterion for this value is the so-called "evolution path." This path contains the correlation of the foregoing generations, which slowly fades out as the generations move on. If the correlation is positive, the solution is expected to be further away, and the path step-size is increased. If the step-size is too large, on the other hand, the following generations tend to cancel each other out. This leads to a negative covariance and the step-size is decreased. This feature is also part of the derandomied ES-DR2-algorithm, while the genetic algorithm has neither step-size adaptation nor covariance matrix adaptation incorporated in it.

\section{RESULTS AND DISCUSSION}

\section{A. R2PI spectrum of 5-methoxyindole}

The R2PI spectrum of 5MOI in the region between the electronic origin $(0,0)$ at 33120.53 and $0,0+1100 \mathrm{~cm}^{-1}$ is shown in Fig. 2. The bands are labeled by their frequencies relative to 0,0 ; bands marked with a dagger have been investigated using rotationally resolved fluorescence excitation spectroscopy. The R2PI spectrum has been intensity corrected to the pulse energy of the dye laser. Comparing the intensities to those in the LIF spectrum of Florio et al. ${ }^{23}$ suggests that our intensities are still influenced by saturation effects and/or by different Franck-Condon (FC) factors of the ionization step, not atypical for a R2PI spectrum. Therefore, these intensities cannot be reliably compared to the Franck-Condon Herzberg-Teller (FCHT) analysis performed in Sec. III B.

\section{B. Calculated vibrational and vibronic spectra}

The $a b$ initio calculated fundamental frequencies of the ground and electronically excited ${ }^{1} L_{b}$ states of anti-5MOI are compiled in Table I. The 54 vibrational modes are grouped

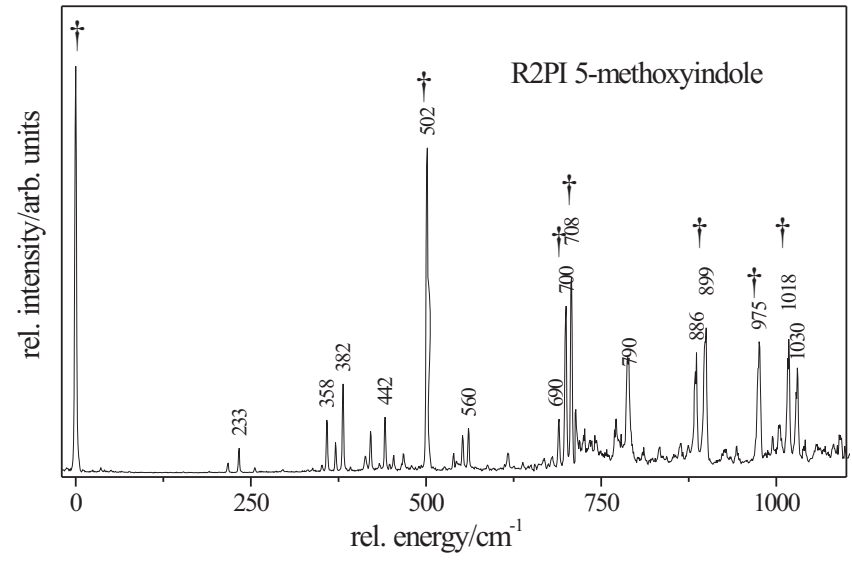

FIG. 2. R2PI spectrum of 5-methoxyindole. Bands that have been investigated with rotational resolution are marked by a dagger.

into 37 in-plane $\left(\mathrm{a}^{\prime}\right)$ modes and 17 out-of-plane ( $\left.\mathrm{a}^{\prime \prime}\right)$ modes. They are given by ascending wavenumbers in Table I and are labeled by their symmetry. $Q_{1}-Q_{37}$ are the in-plane modes and $Q_{38}-Q_{54}$ are the out-of-plane modes. The ground state vibrational wavenumbers are compared to the results of Raman spectroscopy of a polycrystalline 5MOI sample. ${ }^{37}$ For the ground state vibrational wavenumbers, a root mean square deviation (rmsd) of $34 \mathrm{~cm}^{-1}$ from the experimental wavenumbers using unscaled computed wavenumbers is found. Scaling the $\mathrm{CH}$ and $\mathrm{NH}$ stretching vibrations by 0.93 improves the rmsd to $11 \mathrm{~cm}^{-1}$. Assignment of excited state modes to the ground state modes was performed using the elements of the Duchinsky matrix. The largest entries of the complete matrix are given in the last column of Table I. Surprisingly, there is very little mode mixing in 5MOI, compared to indole, where many excited state modes are heavily mixed. Most of the modes in 5MOI retain their identity upon electronic excitation, many of them even retain their energetic position. There are many entries close to one on the diagonal of the Duchinsky matrix, making the assignment of the ${ }^{1} L_{b}$ modes to the respective ground state modes straightforward.

Using the ${ }^{1} L_{b}$ normal modes $Q_{i}$ as basis for the nuclear displacements along which the gradients of the electronic transition dipole moment (TDM) are calculated, ${ }^{17}$ we computed the spectrum of 5MOI in the FCHT approximation, ${ }^{38}$ and compared it to the spectrum computed using the FC approximation (see Fig. 3). At first sight, no difference in intensities can be seen. Also the band type, which reflects the orientation of the transition dipole moment, is practically the same in the FC and the FCHT approximation. This is very different from the cases of indole ${ }^{17,18}$ and tryptamine, ${ }^{39}$ where strong vibronic mixing significantly affects both the intensities and the band type of many vibronic bands.

A preliminary assignment of the vibronic bands in the observed spectrum (Fig. 2) can be made on the basis of the CC2 calculations (see Table I). Although the chromophore in the ${ }^{1} L_{b}$ state is slightly nonplanar, we designate the vibrations as $\mathrm{a}^{\prime}$ and $\mathrm{a}^{\prime \prime}$, as if the symmetry was $\mathrm{C}_{s}$ (with the plane of symmetry in the aromatic plane). Nearly all of the observed bands are of $\mathrm{a}^{\prime}$ symmetry, which would be the only allowed ones in the $\mathrm{C}_{s}$ symmetry. The lowest wavenumber 
TABLE I. Calculated vibrational wavenumbers in the ground and electronically excited ${ }^{1} L_{b}$ states from CC 2 calculations with the cc-pVTZ basis set. The first column gives the mode numbering for the ground state and the last column gives the respective combinations for the ${ }^{1} L_{b}$ state from the Duchinsky analysis (see text for details).

\begin{tabular}{|c|c|c|c|c|c|c|}
\hline \multirow[b]{2}{*}{ Mode } & \multirow[b]{2}{*}{ Sym. } & \multicolumn{2}{|c|}{$S_{0}$} & \multicolumn{2}{|c|}{${ }^{1} L_{b}$} & \multirow[b]{2}{*}{ Duchinsky } \\
\hline & & Calc. & Obs. ${ }^{a}$ & Calc. & Obs. $^{\mathrm{b}}$ & \\
\hline$Q_{54}$ & $\mathrm{a}^{\prime \prime}$ & 76 & $\cdots$ & 77 & & $Q_{54}\left(L_{b}\right)=+0.99 Q_{54}\left(S_{0}\right)$ \\
\hline$Q_{53}$ & $a^{\prime \prime}$ & 140 & 167 & 104 & 109 & $Q_{53}\left(L_{b}\right)=-0.98 Q_{53}\left(S_{0}\right)$ \\
\hline$Q_{52}$ & $a^{\prime \prime}$ & 207 & 208 & 171 & & $Q_{52}\left(L_{b}\right)=+0.87 Q_{52}\left(S_{0}\right)$ \\
\hline$Q_{37}$ & $a^{\prime}$ & 220 & 218 & 227 & 233 & $Q_{37}\left(L_{b}\right)=+0.82 Q_{37}\left(S_{0}\right)$ \\
\hline$Q_{51}$ & $\mathrm{a}^{\prime \prime}$ & 271 & 245 & 194 & 191 & $Q_{51}\left(L_{b}\right)=+0.73 Q_{51}\left(S_{0}\right)$ \\
\hline$Q_{50}$ & $\mathrm{a}^{\prime \prime}$ & 352 & 345 & 440 & & $Q_{47}\left(L_{b}\right)=-0.87 Q_{50}\left(S_{0}\right)$ \\
\hline$Q_{49}$ & $\mathrm{a}^{\prime \prime}$ & 359 & $\cdots$ & 263 & & $Q_{50}\left(L_{b}\right)=-0.74 Q_{49}\left(S_{0}\right)$ \\
\hline$Q_{36}$ & $\mathrm{a}^{\prime}$ & 372 & 370 & 370 & 358 & $Q_{36}\left(L_{b}\right)=-0.91 Q_{36}\left(S_{0}\right)$ \\
\hline$Q_{48}$ & $a^{\prime \prime}$ & 423 & 430 & 278 & & $Q_{49}\left(L_{b}\right)=-0.67 Q_{48}\left(S_{0}\right)+0.41 Q_{50}\left(S_{0}\right)$ \\
\hline$Q_{35}$ & $a^{\prime}$ & 459 & 458 & 423 & 442 & $Q_{35}\left(L_{b}\right)=-0.75 Q_{35}\left(S_{0}\right)$ \\
\hline$Q_{34}$ & $\mathrm{a}^{\prime}$ & 527 & 525 & 510 & 502 & $Q_{34}\left(L_{b}\right)=+0.84 Q_{34}\left(S_{0}\right)$ \\
\hline$Q_{47}$ & $a^{\prime \prime}$ & 574 & 513 & 471 & & $Q_{46}\left(L_{b}\right)=+0.74 Q_{47}\left(S_{0}\right)$ \\
\hline$Q_{46}$ & $a^{\prime \prime}$ & 597 & 603 & 554 & & $Q_{44}\left(L_{b}\right)=-0.63 Q_{46}\left(S_{0}\right)$ \\
\hline$Q_{33}$ & $a^{\prime}$ & 608 & 603 & 572 & 560 & $Q_{33}\left(L_{b}\right)=-0.91 Q_{33}\left(S_{0}\right)$ \\
\hline$Q_{45}$ & $a^{\prime \prime}$ & 678 & 635 & 383 & & $Q_{48}\left(L_{b}\right)=+0.54 Q_{45}\left(S_{0}\right)+0.29 Q_{46}\left(S_{0}\right)$ \\
\hline$Q_{32}$ & $a^{\prime}$ & 751 & 746 & 713 & 700 & $Q_{32}\left(L_{b}\right)=+0.82 Q_{32}\left(S_{0}\right)$ \\
\hline$Q_{44}$ & $a^{\prime \prime}$ & 764 & 770 & 724 & & $Q_{41}\left(L_{b}\right)=-0.79 Q_{44}\left(S_{0}\right)$ \\
\hline$Q_{43}$ & $a^{\prime \prime}$ & 789 & 810 & 500 & & $Q_{45}\left(L_{b}\right)=+0.38 Q_{43}\left(S_{0}\right)+0.51 Q_{47}\left(S_{0}\right)$ \\
\hline$Q_{31}$ & $a^{\prime}$ & 808 & 795 & 798 & 790 & $Q_{31}\left(L_{b}\right)=-0.96 Q_{31}\left(S_{0}\right)$ \\
\hline$Q_{42}$ & $a^{\prime \prime}$ & 819 & 847 & 631 & & $Q_{43}\left(L_{b}\right)=+0.78 Q_{42}\left(S_{0}\right)$ \\
\hline$Q_{30}$ & $\mathrm{a}^{\prime}$ & 906 & 900 & 844 & & $Q_{30}\left(L_{b}\right)=-0.97 Q_{30}\left(S_{0}\right)$ \\
\hline$Q_{41}$ & $a^{\prime \prime}$ & 939 & 932 & 693 & & $Q_{42}\left(L_{b}\right)=-0.64 Q_{41}\left(S_{0}\right)-0.51 Q_{42}\left(S_{0}\right)$ \\
\hline$Q_{29}$ & $\mathrm{a}^{\prime}$ & 954 & 940 & 909 & 899 & $Q_{29}\left(L_{b}\right)=+0.87 Q_{29}\left(S_{0}\right)$ \\
\hline$Q_{28}$ & $\mathrm{a}^{\prime}$ & 1058 & 1035 & 994 & 975 & $Q_{28}\left(L_{b}\right)=+0.79 Q_{28}\left(S_{0}\right)$ \\
\hline$Q_{27}$ & $a^{\prime}$ & 1090 & 1070 & 1017 & 1016 & $Q_{27}\left(L_{b}\right)=-0.85 Q_{27}\left(S_{0}\right)+0.58 Q_{28}\left(S_{0}\right)$ \\
\hline$Q_{26}$ & $a^{\prime}$ & 1112 & 1102 & 1071 & & $Q_{26}\left(L_{b}\right)=-0.79 Q_{26}\left(S_{0}\right)$ \\
\hline$Q_{40}$ & $\mathrm{a}^{\prime \prime}$ & 1124 & 1105 & 926 & & $Q_{40}\left(L_{b}\right)=+0.76 Q_{40}\left(S_{0}\right)$ \\
\hline$Q_{25}$ & $\mathrm{a}^{\prime}$ & 1150 & 1132 & 1104 & & $Q_{25}\left(L_{b}\right)=+0.83 Q_{25}\left(S_{0}\right)$ \\
\hline$Q_{24}$ & $\mathrm{a}^{\prime}$ & 1185 & 1157 & 1163 & & $Q_{24}\left(L_{b}\right)=+0.90 Q_{24}\left(S_{0}\right)$ \\
\hline$Q_{39}$ & $a^{\prime \prime}$ & 1186 & $\cdots$ & 1170 & & $Q_{39}\left(L_{b}\right)=-0.97 Q_{39}\left(S_{0}\right)$ \\
\hline$Q_{23}$ & $a^{\prime}$ & 1231 & 1200 & 1213 & 1212 & $Q_{23}\left(L_{b}\right)=+0.94 Q_{23}\left(S_{0}\right)$ \\
\hline$Q_{22}$ & $\mathrm{a}^{\prime}$ & 1253 & 1235 & 1265 & & $Q_{21}\left(L_{b}\right)=+0.86 Q_{22}\left(S_{0}\right)$ \\
\hline$Q_{21}$ & $\mathrm{a}^{\prime}$ & 1272 & 1255 & 1232 & & $Q_{22}\left(L_{b}\right)=-0.81 Q_{21}\left(S_{0}\right)$ \\
\hline$Q_{20}$ & $a^{\prime}$ & 1319 & 1290 & 1298 & & $Q_{20}\left(L_{b}\right)=+0.83 Q_{20}\left(S_{0}\right)$ \\
\hline$Q_{19}$ & $a^{\prime}$ & 1354 & 1345 & 1314 & & $Q_{19}\left(L_{b}\right)=-0.81 Q_{19}\left(S_{0}\right)$ \\
\hline$Q_{18}$ & $\mathrm{a}^{\prime}$ & 1405 & 1420 & 1361 & 1349 & $Q_{18}\left(L_{b}\right)=+0.67 Q_{18}\left(S_{0}\right)-0.54 Q_{19}\left(S_{0}\right)$ \\
\hline$Q_{17}$ & $\mathrm{a}^{\prime}$ & 1464 & 1440 & 1410 & 1416 & $Q_{17}\left(L_{b}\right)=-0.66 Q_{17}\left(S_{0}\right)+0.53 Q_{15}\left(S_{0}\right)$ \\
\hline$Q_{16}$ & $\mathrm{a}^{\prime}$ & 1487 & 1452 & 1416 & 1457 & $Q_{16}\left(L_{b}\right)=+0.79 Q_{16}\left(S_{0}\right)$ \\
\hline$Q_{15}$ & $\mathrm{a}^{\prime}$ & 1500 & 1483 & 1616 & & $Q_{15}\left(L_{b}\right)=-0.62 Q_{15}\left(S_{0}\right)-0.49 Q_{11}\left(S_{0}\right)$ \\
\hline$Q_{38}$ & $\mathrm{a}^{\prime \prime}$ & 1503 & 1470 & 1504 & & $Q_{38}\left(L_{b}\right)=-0.98 Q_{38}\left(S_{0}\right)$ \\
\hline$Q_{14}$ & $a^{\prime}$ & 1513 & 1509 & 1497 & & $Q_{13}\left(L_{b}\right)=-0.63 Q_{14}\left(S_{0}\right)-0.56 Q_{12}\left(S_{0}\right)$ \\
\hline$Q_{13}$ & $\mathrm{a}^{\prime}$ & 1536 & $\cdots$ & 1526 & & $Q_{11}\left(L_{b}\right)=+0.91 Q_{13}\left(S_{0}\right)$ \\
\hline$Q_{12}$ & $\mathrm{a}^{\prime}$ & 1544 & 1580 & 1465 & & $Q_{14}\left(L_{b}\right)=+0.69 Q_{12}\left(S_{0}\right)-0.35 Q 14$ \\
\hline$Q_{11}$ & $\mathrm{a}^{\prime}$ & 1612 & 1624 & 1510 & & $Q_{12}\left(L_{b}\right)=+0.74 Q_{11}\left(S_{0}\right)$ \\
\hline$Q_{10}$ & $a^{\prime}$ & 1654 & $\cdots$ & 1577 & & $Q_{10}\left(L_{b}\right)=-0.85 Q_{10}\left(S_{0}\right)$ \\
\hline$Q_{9}$ & $a^{\prime}$ & 3048 & 2833 & 3078 & & $Q_{9}\left(L_{b}\right)=-1.00 Q_{9}\left(S_{0}\right)$ \\
\hline$Q_{8}$ & $a^{\prime}$ & 3125 & 2911 & 3171 & & $Q_{8}\left(L_{b}\right)=-1.00 Q_{8}\left(S_{0}\right)$ \\
\hline$Q_{7}$ & $\mathrm{a}^{\prime}$ & 3198 & 2954 & 3215 & & $Q_{7}\left(L_{b}\right)=+1.00 Q_{7}\left(S_{0}\right)$ \\
\hline$Q_{6}$ & $\mathrm{a}^{\prime}$ & 3208 & 2991 & 3223 & & $Q_{6}\left(L_{b}\right)=+1.00 Q_{6}\left(S_{0}\right)$ \\
\hline$Q_{5}$ & $a^{\prime}$ & 3232 & 3028 & 3245 & & $Q_{5}\left(L_{b}\right)=+0.99 Q_{5}\left(S_{0}\right)$ \\
\hline$Q_{4}$ & $a^{\prime}$ & 3240 & 3037 & 3255 & & $Q_{4}\left(L_{b}\right)=+0.99 Q_{4}\left(S_{0}\right)$ \\
\hline$Q_{3}$ & $\mathrm{a}^{\prime}$ & 3274 & 3106 & 3270 & & $Q_{3}\left(L_{b}\right)=+0.93 Q_{3}\left(S_{0}\right)$ \\
\hline$Q_{2}$ & $\mathrm{a}^{\prime}$ & 3294 & 3125 & 3303 & & $Q_{2}\left(L_{b}\right)=+0.93 Q_{2}\left(S_{0}\right)$ \\
\hline$Q_{1}$ & $\mathrm{a}^{\prime}$ & 3665 & 3402 & 3631 & & $Q_{1}\left(L_{b}\right)=-1.00 Q_{1}\left(S_{0}\right)$ \\
\hline
\end{tabular}

${ }^{\mathrm{a}}$ Ground state frequencies from Ref. 37.

"Excited state frequencies from this work; wavenumbers of the forbidden a" vibrations are calculated from the experimental overtones assuming harmonic behavior. 


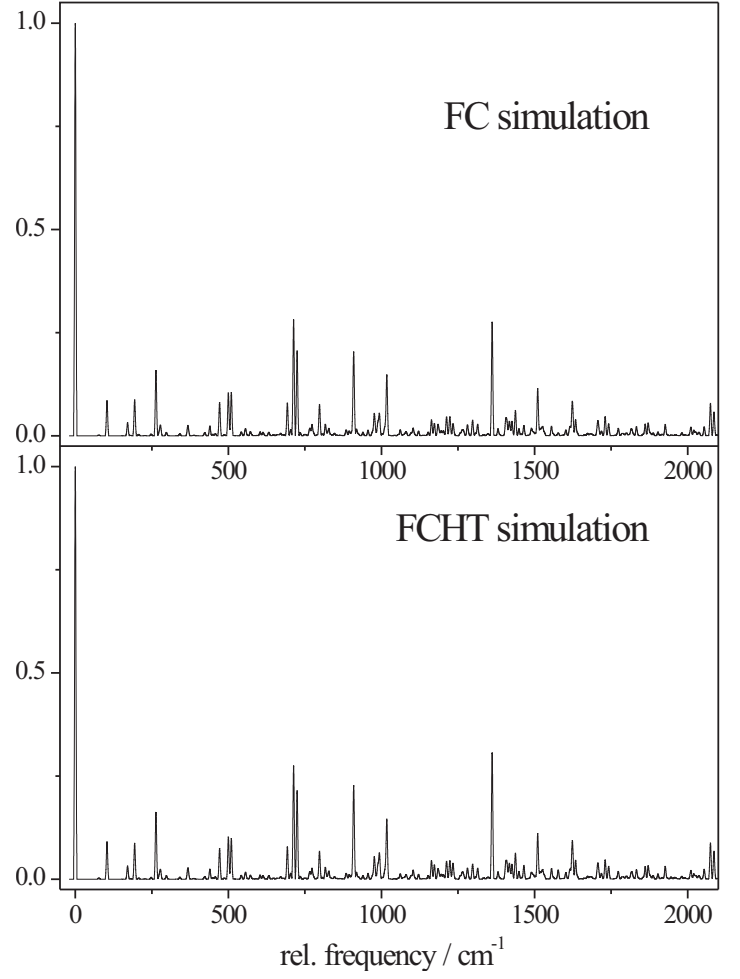

FIG. 3. Comparison of the FC simulation of the 5MOI spectrum with the FCHT simulation.

band is observed at $218 \mathrm{~cm}^{-1}$. It is assigned as the first overtone of the a" vibration $Q_{53}$, calculated at $104 \mathrm{~cm}^{-1}$. This motion can be described as a butterfly vibration of the two rings. The following band at $233 \mathrm{~cm}^{-1}$ is assigned to the first totally symmetric a' vibration $Q_{37}$, calculated at $227 \mathrm{~cm}^{-1}$. The $358 \mathrm{~cm}^{-1}$ band is assigned to vibration $Q_{36}$. The first overtone of the a" vibration $Q_{51}$ is observed at $382 \mathrm{~cm}^{-1}$. Vibration $Q_{35}$, calculated at $423 \mathrm{~cm}^{-1}$, is observed at $442 \mathrm{~cm}^{-1}$. The vibronic band at $502 \mathrm{~cm}^{-1}$ is assigned to $Q_{34}$, which is calculated at $510 \mathrm{~cm}^{-1}$. This vibration has mainly the character of a $\mathrm{C}-\mathrm{O}-\mathrm{C}$ bending vibration $Q_{33}$, which will become important in later discussion. $Q_{33}$ is observed at $560 \mathrm{~cm}^{-1}$. If the energetically following band at $700 \mathrm{~cm}^{-1}$ is assigned to $Q_{32}$, there are no other a' vibrations sufficiently close to explain the $708 \mathrm{~cm}^{-1}$ band. We therefore propose a Fermi resonance between $Q_{32}$ and the combination of $Q_{50}$ and $Q_{49}$ (both a") to be responsible for the pair of bands at $700 / 708 \mathrm{~cm}^{-1}$. The $790 \mathrm{~cm}^{-1}$ band can be assigned to the following a' vibration $Q_{31}, 899 \mathrm{~cm}^{-1}$ to $Q_{29}$, $975 \mathrm{~cm}^{-1}$ to $Q_{28}$, and $1016 \mathrm{~cm}^{-1}$ to $Q_{27}$.

\section{High resolution spectrum of the origin band of 5-methoxyindole}

Figure 4 shows the rotationally resolved spectrum of the electronic origin of 5MOI along with a simulation using the best parameters from the CMA-ES fit given in Table II. The spectrum was fit to an asymmetric rotor Hamiltonian with axis reorientation ${ }^{40,41}$ using a derandomized evolutionary strategy. ${ }^{35}$ The uncertainties of the parameters are given in parentheses and are obtained as standard deviations by performing a quantum number assigned fit. In this fit, those

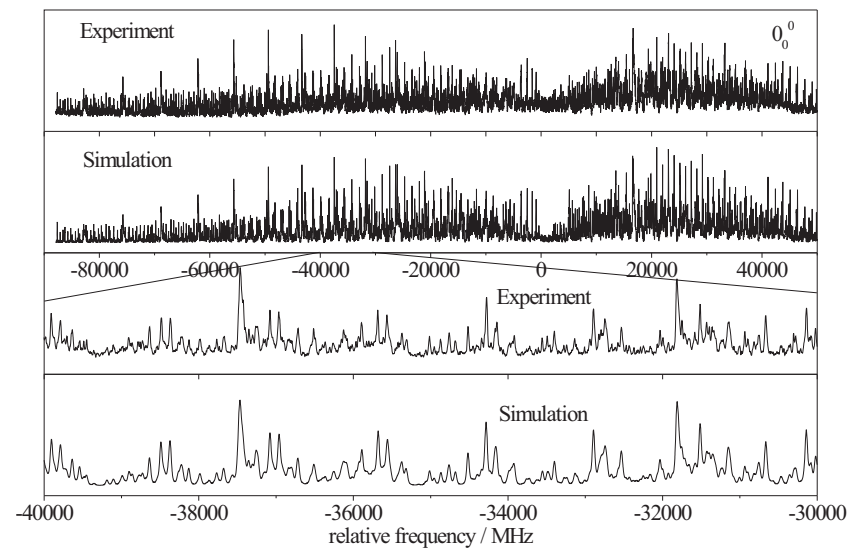

FIG. 4. Rotationally resolved electronic spectrum of the electronic origin of 5-methoxyindole. The second trace shows the simulation using the best parameters from Table II. The lowest traces show expanded scale portions of the experimental spectrum and the corresponding simulation.

transitions are included that would be used in a typical manual assignment of the spectrum; that is, transitions with an intensity larger than $5 \%$ of the maximum intensity and with rotational quantum numbers up to $\mathrm{J}=10$.

Comparison of the rotational constants to the results of the $a b$ initio calculations immediately shows that the observed band is due to absorption of the anti-conformer, as proposed by Florio, et al. ${ }^{23}$ Both the structures in the ground state, as well as the changes of the structure upon excitation to the $L_{b}$ state, are well reproduced using $\mathrm{CC} 2$ with the ccpVTZ basis set (see below). The nonzero inertial defect of $-3.59 \mathrm{amu} \AA^{2}$ in the electronic ground state and of $-3.73 \mathrm{amu} \AA^{2}$ in the excited state has its origin in the hydrogen atoms of the methyl group, which are not in the aromatic plane. The CC2/cc-pVTZ calculated inertial defects of the ground and excited state equilibrium structures were determined to be -3.44 and -3.62 amu $\AA^{2}$, respectively. The band was found to be an $a b$ hybrid, with $72 \% b$ - and $28 \% a$-type character. This corresponds to an in-plane angle $\theta$ of the TDM with the inertial $a$ axis of $57^{\circ}$. From comparison to the theoretical TDM orientation (see below) and to the results for indole, the sign of $\theta$ is determined to be positive. The axis reorientation angle $\theta_{T}$ is determined to be $0.4^{\circ}$. Its sign is determined to be equal to that of the TDM angle $\theta$, in good agreement with the theoretical predictions. The lifetime of the vibrationless origin has been determined from a Lorentz contribution of $23.7 \mathrm{MHz}$ to the Voigt profile to be 6.7 ns.

Geometric parameters of syn- and anti-5MOI in their electronic ground state and lowest two excited singlet states from CC2/cc-pVTZ calculations are given in Table S1 of the online supporting material. ${ }^{46}$ Contrary to the case of indole, ${ }^{17,18}$ all excited state structures were identified as minima on the potential energy surface (PES) from a normal mode analysis at the level of structure optimization. From the optimized geometries, the rotational constants for both conformers in the three singlet states were determined. They are given in Table III. For comparison, the experimentally determined rotational constants of the origin band also are listed there. Both the ground state values and the changes of 
TABLE II. Molecular parameters from the fits to the rotationally resolved electronic spectra of several vibronic bands of 5-methoxyindole.

\begin{tabular}{|c|c|c|c|c|c|c|c|c|c|}
\hline & Origin & 502 (red) & 502 (blue) & 700 & 708 (red) & 708 (blue) & 899 (red) & 899 (blue) & 1016 \\
\hline$A^{\prime \prime}(\mathrm{MHz})$ & $2851.29(1)$ & $2851.29(1)$ & 2851.29(1) & $2851.29(1)$ & $2851.29(1)$ & 2851.29(1) & 2851.29(1) & $2851.29(1)$ & 2851.29(1) \\
\hline$B^{\prime \prime}(\mathrm{MHz})$ & $757.72(1)$ & $757.72(1)$ & 757.72(1) & 757.72(1) & $757.72(1)$ & 757.72(1) & 757.72(1) & 757.72(1) & 757.72(1) \\
\hline$C^{\prime \prime}(\mathrm{MHz})$ & $601.21(1)$ & $601.21(1)$ & $601.21(1)$ & $601.21(1)$ & $601.21(1)$ & $601.21(1)$ & $601.21(1)$ & 601.21(1) & 601.21(1) \\
\hline$\Delta I^{\prime \prime}\left(\mathrm{amu} \AA^{2}\right)^{\mathrm{a}}$ & $-3.59(3)$ & $-3.59(3)$ & $-3.59(3)$ & $-3.59(3)$ & $-3.59(3)$ & $-3.59(3)$ & $-3.59(3)$ & $-3.59(3)$ & $-3.59(3)$ \\
\hline$\Delta A(\mathrm{MHz})^{\mathrm{b}}$ & $-99.32(1)$ & $-96.46(1)$ & $-96.46(1)$ & $-99.38(1)$ & $-98.72(1)$ & $-99.65(1)$ & $-105.14(1)$ & $-99.45(1)$ & $-102.89(1)$ \\
\hline$\Delta B(\mathrm{MHz})^{\mathrm{b}}$ & $+1.26(1)$ & $+0.96(1)$ & $+0.96(1)$ & $+1.05(1)$ & $+0.67(1)$ & $+1.07(1)$ & $+0.97(1)$ & $+0.90(1)$ & $+1.13(1)$ \\
\hline$\Delta C(\mathrm{MHz})^{\mathrm{b}}$ & $-3.63(1)$ & $-3.92(1)$ & $-3.92(1)$ & $-3.61(1)$ & $-3.47(1)$ & $-3.58(1)$ & $-3.50(1)$ & $-3.70(1)$ & $-3.72(1)$ \\
\hline$\Delta I^{\prime}\left(\mathrm{amu} \AA^{2}\right)^{\mathrm{a}}$ & $-3.73(2)$ & $-3.46(2)$ & $-3.46(2)$ & $-3.98(2)$ & $-4.45(2)$ & $-4.04(2)$ & $-4.44(2)$ & $-4.00(2)$ & $-4.03(2)$ \\
\hline$\theta(\mathrm{deg})$ & $\pm 58.4(1)$ & $\pm 55.2(1)$ & $\pm 55.2(1)$ & $\pm 62.5(2)$ & $\pm 63.7(2)$ & $\pm 63.9(2)$ & $\pm 64.6(1)$ & $\pm 63.9(1)$ & $\pm 62.3(1)$ \\
\hline$\theta_{T}(\mathrm{deg})$ & $\pm 0.40(5)$ & & & & & & & & \\
\hline$\phi(\mathrm{deg})$ & 90 & 90 & 90 & 90 & 90 & 90 & 90 & 90 & 90 \\
\hline$\mu_{a}^{2}$ & 0.28 & 0.33 & 0.33 & 0.20 & 0.22 & 0.19 & 0.21 & 0.26 & 0.30 \\
\hline$\mu_{b}^{2}$ & 0.72 & 0.67 & 0.67 & 0.80 & 0.78 & 0.81 & 0.79 & 0.74 & 0.70 \\
\hline$\mu_{c}^{2}$ & 0.00 & 0.00 & 0.00 & 0.00 & 0.00 & 0.00 & 0.00 & 0.00 & 0.00 \\
\hline$\nu_{0}\left(\mathrm{~cm}^{-1}\right)$ & $33120.53(1)$ & $33622.61(1)$ & $33622.61(1)$ & $33820.55(1)$ & $33829.00(1)$ & $33829.04(1)$ & $34019.05(1)$ & $34019.06(1)$ & $34173.15(1)$ \\
\hline$\Delta \nu_{0}\left(\mathrm{~cm}^{-1}\right)$ & $\cdots$ & $502.08(1)$ & $502.08(1)$ & $700.02(1)$ & $708.47(1)$ & $708.51(1)$ & $898.52(1)$ & $898.53(1)$ & 1016.62(1) \\
\hline$t_{1 / 2}(\mathrm{~ns})$ & 6.7 & 9.4 & 9.4 & 2.6 & 5.4 & 5.4 & 5.0 & 5.0 & $\cdots$ \\
\hline
\end{tabular}

The inertial defect $\Delta I$ is defined as: $\Delta I=I_{c}-I_{b}-I_{a}$, where the $I_{g}$ are the moments of inertia with respect to the main inertial axes $g=a, b, c$.

${ }^{\mathrm{b}}$ Changes of the rotational constants are defined as: $\Delta B_{g}=B_{g}^{\prime}-B_{g}^{\prime \prime}$, with $B_{g}$ as rotational constants with respect to the inertial axes $g=a, b, c$.

the rotational constants upon excitation prove that the observed conformer is anti-5MOI.

A geometry change upon electronic excitation might cause a rotation of the inertial axes of the molecule, which can be described for a planar molecule using a single rotational angle $\theta_{T}$. Since the oxygen of the methoxy group is located in the indole plane and the methyl group is a symmetric (internal) rotor, we can treat 5MOI as planar molecule. The $c$-axis, which is perpendicular to the aromatic plane, coincides for the ground and excited states, and $\theta_{T}$ describes the rotation of the $a^{\prime \prime}$-axis of the ground state into the $a^{\prime}$-axis of the excited state about the $c$-axis. This effect of axis reorientation on rotationally resolved electronic spectra has been described in detail elsewhere. ${ }^{40}$

From the $a b$ initio optimized structures, the angle of reorientation of the inertial axis system upon electronic excitation $\theta_{T}$ can be determined using the relation for planar molecules given by Hougen and Watson ${ }^{40,41}$

$$
\tan \left(\theta_{T}\right)=\frac{\sum_{i} m_{i}\left(a_{i}^{\prime} b_{i}^{\prime \prime}-b_{i}^{\prime} a_{i}^{\prime \prime}\right)}{\sum_{i} m_{i}\left(a_{i}^{\prime} a_{i}^{\prime \prime}+b_{i}^{\prime} b_{i}^{\prime \prime}\right)} .
$$

Here, the doubly primed coordinates refer to the principal axis system in the electronic ground state and the singly primed quantities refer to the respective excited state inertial system; the $m_{i}$ are the atomic masses. Using the CC2 optimized structures for the $S_{0}$ and ${ }^{1} L_{b}$ states, we obtained a reorientation angle of $+0.2^{\circ}$ for the ${ }^{1} L_{b}$ state. A positive angle is defined by a clockwise rotation of the ground state inertial axes into the excited state axes.

Table IV gives the calculated energy differences of the syn- and anti-conformers of 5MOI at the CC2 and DFT/ MRCI levels of theory. The anti-conformer is by $464 \mathrm{~cm}^{-1}$ lower in energy at the CC2 level and by $359 \mathrm{~cm}^{-1}$ using the DFT/MRCI approach. The relative stabilization energies given in Table IV contain zero-point energy corrections at the CC2/cc-pVTZ level of theory. Depending on the height of the barrier separating the two conformers, it should be possible to observe both conformers in a molecular beam experiment. Nevertheless, in our experiments we were able only to observe the anti-conformer. We evaluated the barrier to interconversion of the syn- and anti-conformers at the MP2/6-311G(d,p) level to be $1300 \mathrm{~cm}^{-1}$. This barrier is strongly coupled to the methyl torsional motion in the methoxy group (see below).

Table IV gives the adiabatic and vertical excitation and emission energies of syn- and anti-5MOI, computed at the CC2 level with the cc-pVTZ basis and using DFT/MRCI with the BH-LYP functional on the $\mathrm{CC} 2$ optimized struc-

TABLE III. Rotational constants (in MHz) and inertial defects (in amu $\AA^{2}$ ) of 5MOI in the electronic ground state and their changes upon electronic excitation to ${ }^{1} L_{b}$ and ${ }^{1} L_{a}$ states, computed from the CC2 optimized structures using the cc-pVTZ basis set.

\begin{tabular}{|c|c|c|c|c|c|c|c|c|c|c|c|c|c|}
\hline & \multicolumn{10}{|c|}{$\mathrm{CC} 2$} & \multicolumn{3}{|c|}{ Expt. } \\
\hline & \multicolumn{5}{|c|}{ syn } & \multicolumn{5}{|c|}{ anti } & \multirow[b]{2}{*}{$S_{0}$} & \multirow[b]{2}{*}{$S_{1}$} & \multirow[b]{2}{*}{$S_{1}-S_{0}$} \\
\hline & $S_{0}$ & ${ }^{1} L_{b}$ & ${ }^{1} L_{a}$ & $\Delta{ }^{1} L_{b}$ & $\Delta{ }^{1} L_{a}$ & $S_{0}$ & ${ }^{1} L_{b}$ & ${ }^{1} L_{a}$ & $\Delta{ }^{1} L_{b}$ & $\Delta{ }^{1} L_{a}$ & & & \\
\hline$A$ & 3405 & 3304 & 3354 & -101 & -51 & 2843 & 2735 & 2844 & -108 & +1 & 2851.29 & 2750.97 & -99.32 \\
\hline$B$ & 704 & 703 & 697 & -1 & -7 & 759 & 760 & 747 & +1 & -12 & 757.72 & 758.98 & +1.26 \\
\hline$C$ & 585 & 582 & 579 & -4 & -6 & 601 & 597 & 594 & -4 & -7 & 601.21 & 597.59 & -3.63 \\
\hline$\Delta I$ & -3.36 & -3.45 & -3.67 & $\cdots$ & $\cdots$ & -3.44 & -3.62 & -4.14 & $\cdots$ & $\cdots$ & -3.59 & -3.73 & $\cdots$ \\
\hline
\end{tabular}


TABLE IV. Relative energies and transition moment orientations of the synand anti-conformers of 5MOI and excitation and emission energies to and from the ${ }^{1} L_{b}$ and ${ }^{1} L_{a}$ states. All energies are given in reciprocal centimeters, angles in degrees. The adiabatic excitation energies contain zero-point energy corrections at the $\mathrm{CC} 2$ level.

\begin{tabular}{lccccc}
\hline \hline & \multicolumn{2}{c}{ CC2 } & & \multicolumn{2}{c}{ DFT/MRCI } \\
\cline { 2 - 3 } \cline { 5 - 6 } \cline { 5 - 6 } & syn & anti & & syn & anti \\
\hline Relative energy & 464 & 0 & & 359 & 0 \\
${ }^{1} L_{b} \leftarrow S_{0}$ (adiabatic) & 32852 & 33702 & & 32294 & 32946 \\
$\theta\left({ }^{1} L_{b} \leftarrow S_{0}\right)$ & +66 & +66 & & +68 & +67 \\
${ }^{1} L_{a} \leftarrow S_{0}$ (adiabatic) & 37992 & 38353 & & 36716 & 37233 \\
$\theta\left({ }^{1} L_{a} \leftarrow S_{0}\right)$ & -16 & -16 & & -21 & -22 \\
${ }^{1} L_{b} \leftarrow S_{0}$ (vertical) & 35784 & 36688 & & 34577 & 35239 \\
${ }^{1} L_{a} \leftarrow S_{0}$ (vertical) & 41633 & 42298 & & 39536 & 40108 \\
$S_{0} \leftarrow{ }^{1} L_{b}$ (vertical) & 32595 & 33276 & & 31423 & 31887 \\
$S_{0} \leftarrow{ }^{1} L_{a}$ (vertical) & 34284 & 35062 & & 34233 & 34284 \\
$\Delta\left(L_{a} L_{b}\right)$ (adiabatic) & 4915 & 4651 & & 4197 & 4287 \\
\hline \hline
\end{tabular}

tures. All adiabatic excitation energies contain zero-pointenergy corrections at the $\mathrm{CC} 2$ level. The experimentally observed electronic origin of the anti-conformer at $33120 \mathrm{~cm}^{-1}$ is well reproduced at the DFT/MRCI level (32 $\left.946 \mathrm{~cm}^{-1}\right)$. Both for the syn- and anti-conformers, a ${ }^{1} L_{a} /{ }^{1} L_{b}$ gap of more than $4000 \mathrm{~cm}^{-1}$ is calculated. The origin of the (up to now unobserved) syn-conformer can be expected to be shifted by approximately $650 \mathrm{~cm}^{-1}$ to the red of the anti-origin.

Additionally, the angles $\theta$ between the TDM and the inertial $a$ axis, obtained at both levels of theory, are given in the table. The $\mathrm{CC} 2$ values have been calculated from the average of the Cartesian components of the left and right transition moments. For both syn- and anti-conformers, a positive angle $\theta$ is found for the ${ }^{1} L_{b}$ state and a negative angle for the ${ }^{1} L_{a}$ state. These orientations are similar to the orientation of the ${ }^{1} L_{b}$ TDM orientation in the indole chromophore itself (cf. Fig. 5). The calculated values of $+66^{\circ}$ and $+67^{\circ}$ at the CC2 and DFT/MRCI levels, respectively, are in good agreement with the experimental value of $+58.4^{\circ}$ (cf. Table II).
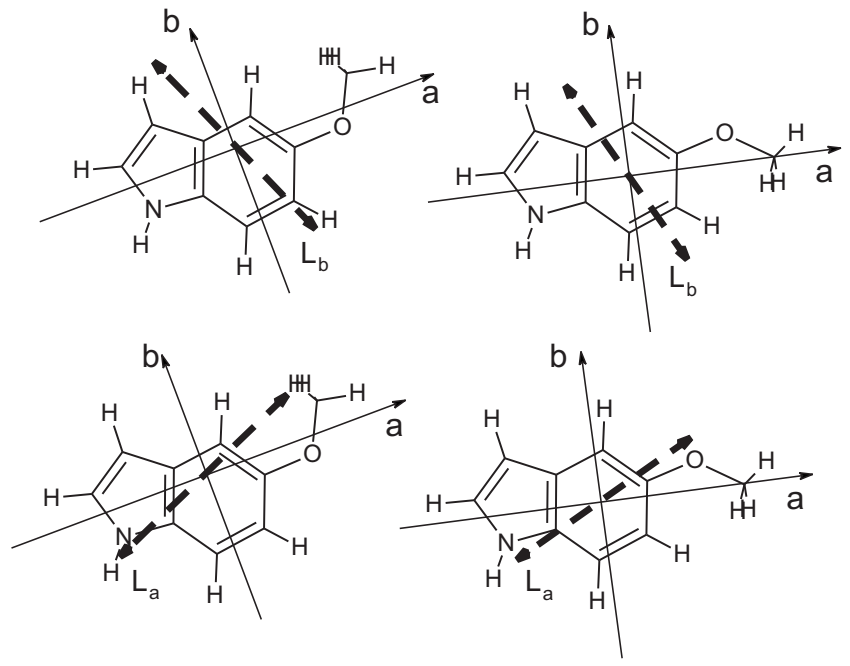

FIG. 5. TDM orientation of the ${ }^{1} L_{b}$ (upper structures) and ${ }^{1} L_{a}$ (lower structures) states of anti- (left structures) and syn-5MOI (right structures).

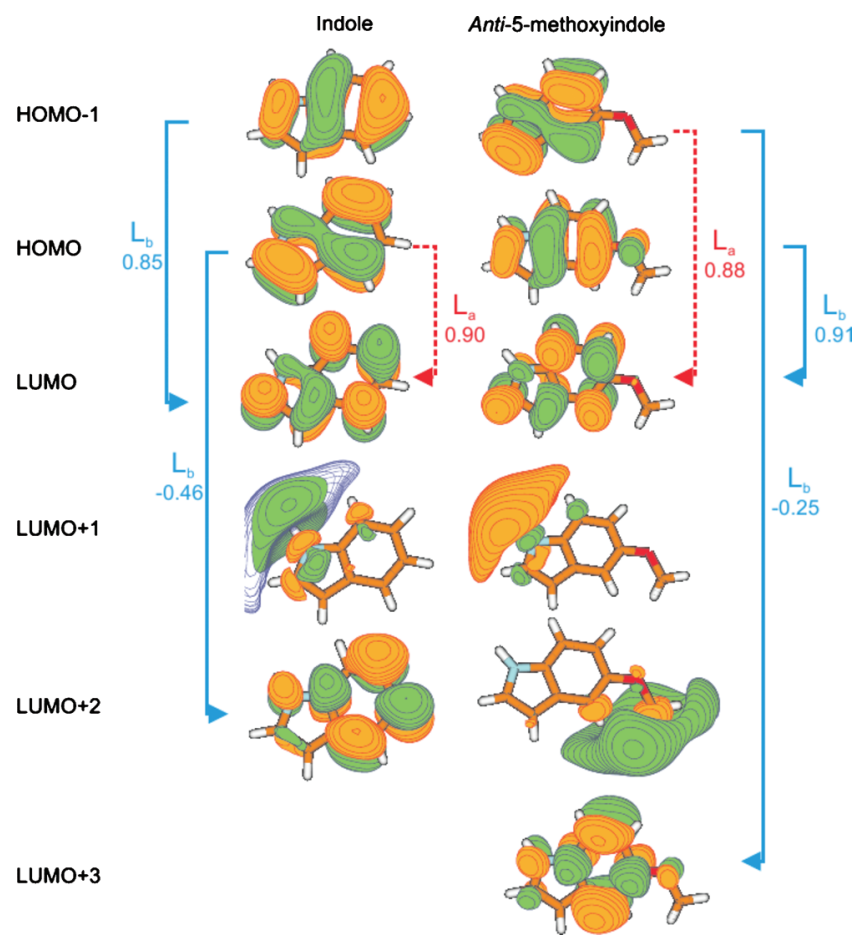

FIG. 6. Frontier orbitals of indole and 5-methoxyindole.

The inertial axis systems of the two conformers are tilted in the $a b$ plane with respect to each other by an angle of $13^{\circ}$. Therefore, the orientation of the TDM in the molecular frame is not the same for the syn- and anti-conformers, although the numbers in Table IV imply that it is. Projecting the TDM orientation of the ${ }^{1} L_{b}$ state of anti-5MOI onto the pseudo- $\mathrm{C}_{2}$ axis in indole, we find an angle of $+45^{\circ}$ compared to $+38^{\circ}$ for the TDM orientation in indole, while for the synconformer, an angle of $+55^{\circ}$ of the TDM with respect to the pseudo- $\mathrm{C}_{2}$ axis in indole is found. The sign of $\theta$ is the same as the sign of $\theta_{T}$.

A closer look at the frontier orbitals that are involved in the two electronic transitions to the ${ }^{1} L_{a}$ and ${ }^{1} L_{b}$ states reveals that the main contribution to the ${ }^{1} L_{b}$ transition is the excitation from the highest occupied molecular orbital to the lowest unoccupied molecular orbital (HOMO $\rightarrow$ LUMO) This is different from the case of indole, where this excitation is the signature for ${ }^{1} L_{a}$ excitation. The reason for this apparent change of orbital ordering can be seen in Fig. 6 from the comparison to the indole frontier orbitals. The methoxy substituent introduces an additional node in the molecular orbital, which was formerly the HOMO-1 in indole, and which now becomes the HOMO in 5MOI. This causes a change in energy ordering between indole and 5MOI for the HOMO and HOMO-1, respectively.

Generally, it is believed that excitation to the ${ }^{1} L_{b}$ state in indole arises from a linear combination of HOMO-1 $\rightarrow$ LUMO and $\mathrm{HOMO} \rightarrow \mathrm{LUMO}+1$ from analogy to the case of catacondensed aromatics such as naphthalene, ${ }^{16,42}$ for which the nomenclature of ${ }^{1} L_{a}$ and ${ }^{1} L_{b}$ was introduced by Platt. ${ }^{15}$ In systems of lower symmetry than $\mathrm{D}_{2 h}$, not only does the meaning of ${ }^{1} L_{a}$ and ${ }^{1} L_{b}$ becomes obscure, but other orbitals might be involved, such as the Rydberg-like orbitals at the indolic $\mathrm{N}-\mathrm{H}$ group or at the methoxy group in $5 \mathrm{MOI}$. 


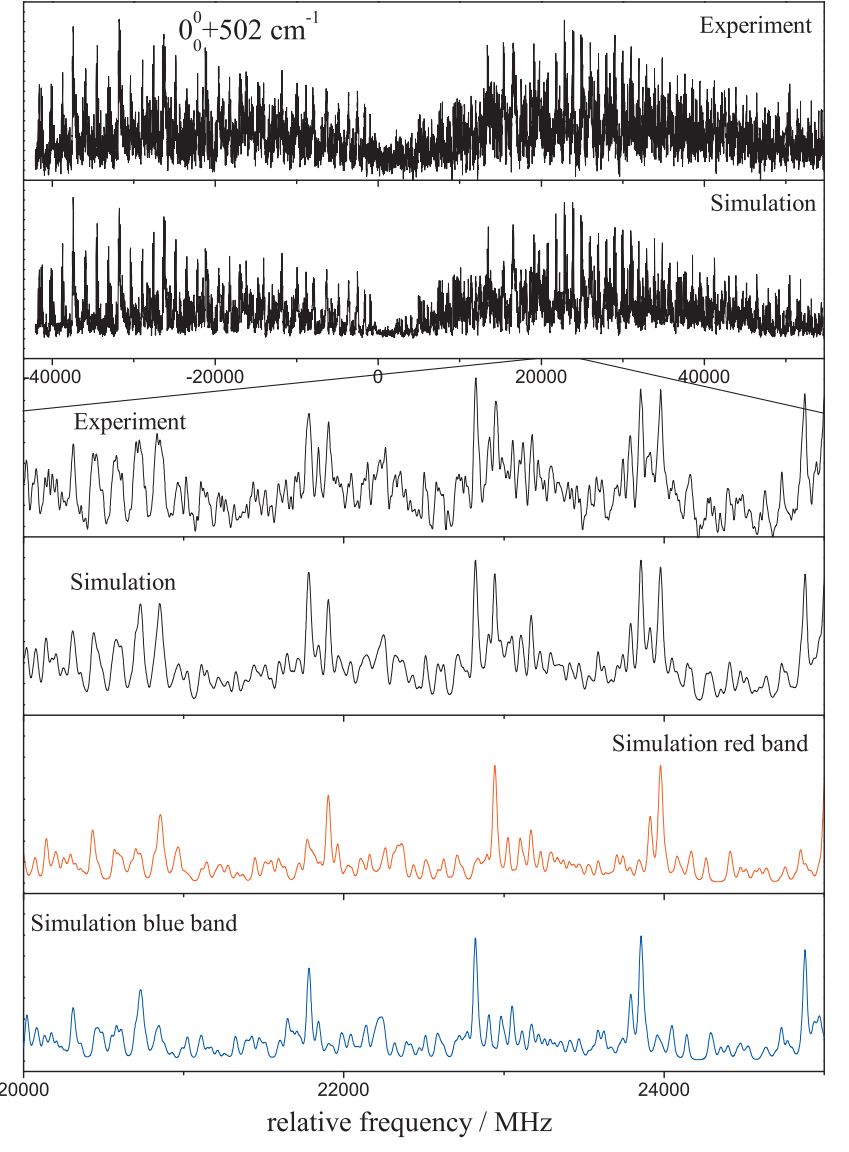

FIG. 7. Rotationally resolved electronic spectrum of the $502 \mathrm{~cm}^{-1}$ band of 5-methoxyindole. The second trace shows the simulation using the best-fit parameters from Table II. The lowest two traces show expanded portions of the experimental spectrum and the simulation.

At the CC2 level with a cc-pVTZ basis, the Rydberg-like $\mathrm{N}-\mathrm{H}$ orbital in indole becomes energetically the LUMO+1 so that the transition to the ${ }^{1} L_{b}$ state now involves the $\mathrm{HOMO}-1 \rightarrow$ LUMO and $\mathrm{HOMO} \rightarrow$ LUMO +2 excitation. Additionally, the Rydberg-like orbital at the methoxy group also lies in the HOMO/LUMO region so that the ${ }^{1} L_{b}$ state here is comprised of HOMO-1 $\rightarrow$ LUMO and HOMO $\rightarrow$ LUMO +3 excitations.

\section{Rotationally resolved spectra of higher vibronic bands}

Figures 7-9 show rotationally resolved electronic spectra of the $0,0+502,700$, and $708 \mathrm{~cm}^{-1}$ bands of 5MOI. The spectra of the +899 and $+1016 \mathrm{~cm}^{-1}$ bands are shown in the supporting online material. ${ }^{46}$ In all cases, the signal-to-noise ratio is not sufficient for a reliable determination of the axis reorientation angle $\theta_{T}$. This is due to the fact that the intensity variations due to axis reorientation are small.

The $502 \mathrm{~cm}^{-1}$ band is composed of two subbands that are separated by $123 \mathrm{MHz}$. The lowest two traces of Fig. 7 show the simulations of the individual components. The molecular parameters that were used in the simulations of the spectra have been obtained from ES fits. The ground state parameters have been set equal to the values of the fit of the origin band. The lifetime determined from the Lorentzian contribution to the Voigt profile is 10.4 ns. All molecular

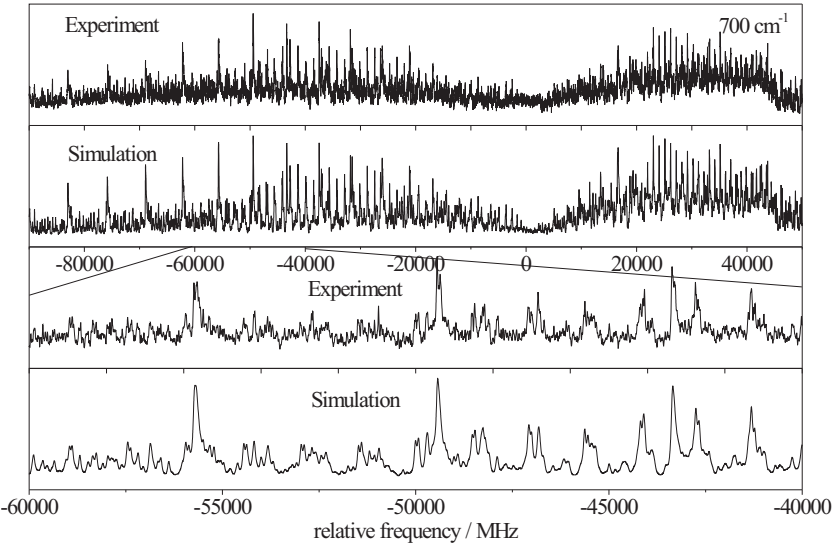

FIG. 8. Rotationally resolved electronic spectrum of the $700 \mathrm{~cm}^{-1}$ band of 5-methoxyindole. The second trace shows the simulation using the best-fit parameters from Table II. The lowest traces show expanded portions of the experimental spectrum and the simulation.

parameters obtained in the fit are compiled in Table II. The orientation of the transition dipole moment of both components is the same as that of the origin band.

Changes of the rotational constants upon vibronic excitation of the $700 \mathrm{~cm}^{-1}$ band (Fig. 8) are very similar to those of the $502 \mathrm{~cm}^{-1}$ band. Also the transition moment orientation of the band at $700 \mathrm{~cm}^{-1}$ is very similar to that of the origin band and the $502 \mathrm{~cm}^{-1}$ band. In contrast to the $502 \mathrm{~cm}^{-1}$ band, the $700 \mathrm{~cm}^{-1}$ band consists of only one

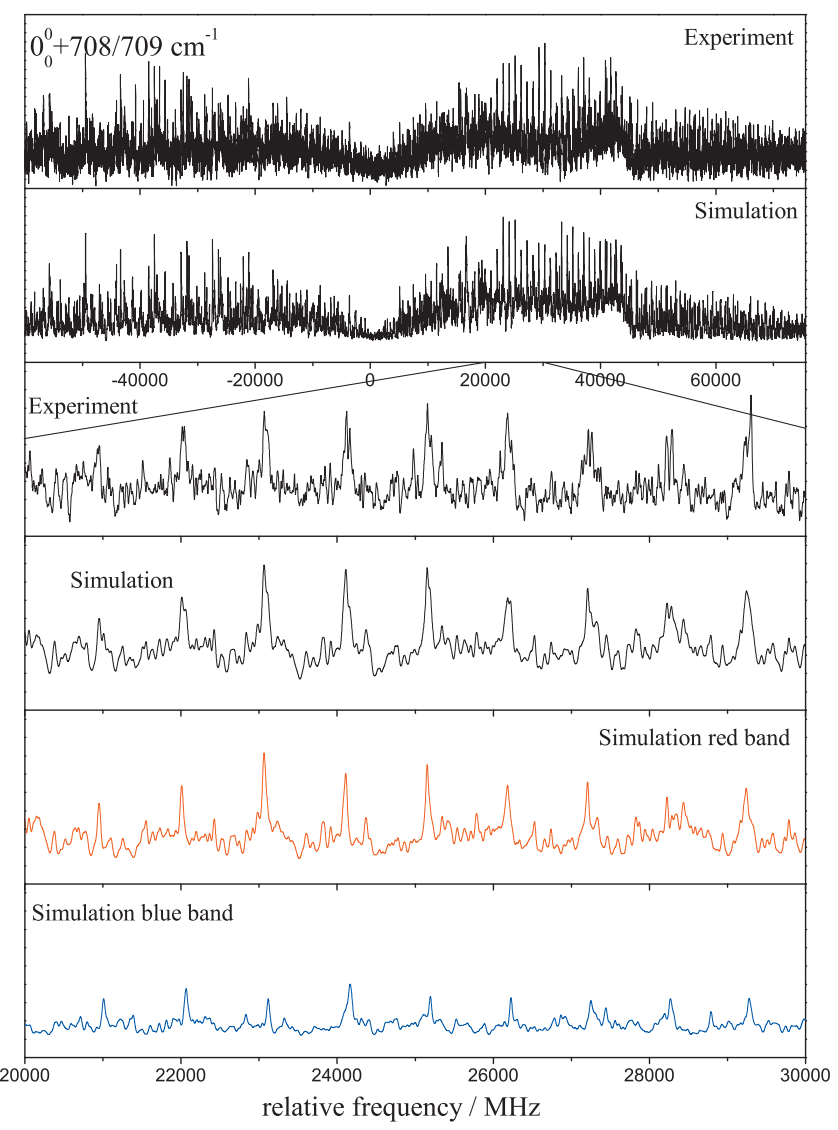

FIG. 9. Rotationally resolved electronic spectrum of the $708 \mathrm{~cm}^{-1}$ band of 5-methoxyindole. The second trace shows the simulation using the best-fit parameters from Table II. The lowest traces show expanded portions of the experimental spectrum and the simulation. 
component within an uncertainty of about $20 \mathrm{MHz}$. The lifetime of this band is determined to be $2.4 \mathrm{~ns}$ from the Lorentzian contribution to the Voigt profile.

The $708 \mathrm{~cm}^{-1}$ band (Fig. 9) is again split into two components, such as the $502 \mathrm{~cm}^{-1}$ band, with a splitting of 1026 MHz. Both components exhibit very similar changes of the rotational constants upon electronic excitation. Also, the vibronic band at $899 \mathrm{~cm}^{-1}$ is a superposition of two vibronic bands, separated by $250 \mathrm{MHz}$. Both components exhibit quite different changes of the rotational constants upon vibronic excitation and are not as easily detected in a autocorrelation of the experimental spectrum as for the $502 \mathrm{~cm}^{-1}$ band.

The highest vibronic band studied here is at $1016 \mathrm{~cm}^{-1}$. Its spectrum is comprised of two subbands, as in the case of the 502 and $899 \mathrm{~cm}^{-1}$ bands. Unfortunately, the second component could not be simulated. It is not clear if it is due to another vibronic band, which coincides exactly and exhibits different rotational constants, or if this band is affected by a strong, hitherto unexplained perturbation.

\section{E. The splitting in the vibronic bands}

The reasons for the splitting of selected vibronic bands into two components are not quite clear. Several explanations might apply here. One possibility is a tunneling splitting due to a hindered internal motion. Two internal rotations are possible in 5MOI. The first is the twofold torsion, which interconverts the anti- and syn-conformers. Since both minima are different, this motion cannot be responsible for the splitting. The second possible motion is the threefold torsion of the methyl rotor in the methoxy group. The $\mathrm{V}_{3}$ torsion of a methoxy group about the $\mathrm{C}-\mathrm{O}$ bond has a very high barrier, at least in the electronic ground state. For anisole (methoxybenzene), a $\mathrm{V}_{3}$ barrier of $1847 \mathrm{~cm}^{-1}$ has been determined from Raman spectroscopy of anisole and anisole- $\mathrm{d}_{3} .{ }^{43}$ The ground state tunneling $\mathrm{A} / \mathrm{E}$ splitting from this barrier would amount to only $0.003 \mathrm{MHz}$, assuming a torsional constant $F$ of $156 \mathrm{GHz}$, typical for a methyl group. If the splitting of $123 \mathrm{MHz}$ observed in the $502 \mathrm{~cm}^{-1}$ band is a consequence of the threefold torsion of the methoxy group, it must therefore be due to the excited state splitting. Selection rules for the torsional transitions are $\mathrm{A} \leftrightarrow \mathrm{A}$ and $\mathrm{E} \leftrightarrow \mathrm{E}$. The experimentally observed splitting of $123 \mathrm{MHz}$ corresponds to a $\mathrm{V}_{3}$ barrier in the excited state of $450 \mathrm{~cm}^{-1}$. Since no torsional splitting is observed in the spectrum of the electronic origin, the reason for the large reduction of the barrier cannot be an electronic one alone, but must be caused by a reduction of an effective barrier due to excitation of the torsional vibration in the excited state, or excitation of a band which is at least strongly coupled to the torsion.

To further elucidate this aspect, we calculated the ground state barrier of 5MOI by varying the dihedral angles defining the torsional angle of the methyl group and relaxing all other molecular parameters at each torsional angle. For a torsional angle of zero, the molecule is in its equilibrium position, which before has been determined to be planar. When scanning the torsional angle, the methoxy group becomes nonplanar with respect to the indole plane. Thus, the minimum

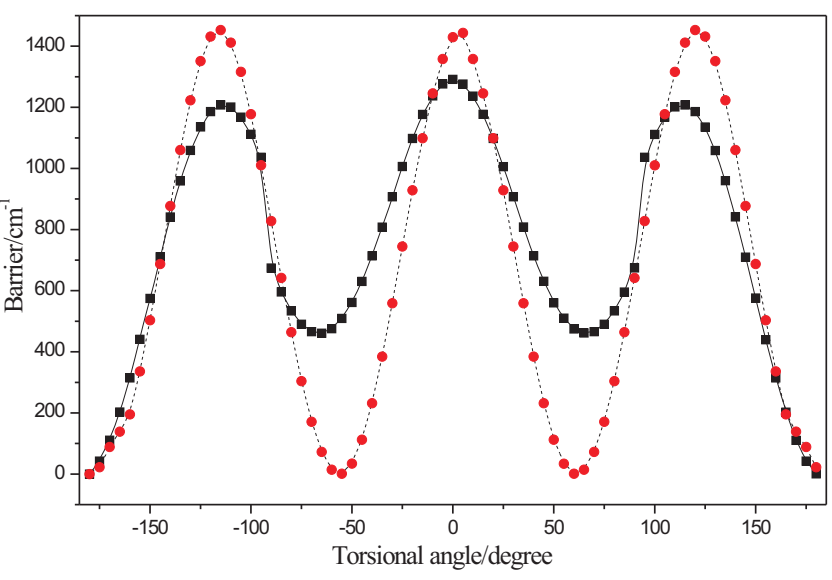

FIG. 10. MP2/6-311G(d,p) torsional barriers of 5MOI. The full line gives the fully relaxed potential energy along the torsional coordinate $\alpha$, the broken line the potential energy along $\alpha$, with the methoxy group held coplanar with the indole ring. For details, see text.

energy path for the methyl $\left(\mathrm{V}_{3}\right)$ torsion is strongly coupled to the methoxy $\left(\mathrm{V}_{2}\right)$ torsional motion. The effective barrier along this path amounts to $1200 \mathrm{~cm}^{-1}$ at the MP2/6$311 \mathrm{G}(\mathrm{d}, \mathrm{p})$ level of theory. At the top of the barrier at $-120^{\circ}$, the methoxy group is rotated out of the plane by $45^{\circ}$. At a torsional angle of $-60^{\circ}$, the methoxy group has swung back into the indole plane, but is on the $s y n$-side. Thus, the energy difference of the minima at -180 and $-60^{\circ}$ gives the energy difference of the syn- and anti-conformers at the MP2/6$311 \mathrm{G}(\mathrm{d}, \mathrm{p})$ level of theory.

Figure 10 shows the completely relaxed energy path, along the torsional coordinate (solid line). Additionally, we calculated a relaxed energy path, in which the methoxy group was kept fixed at a coplanar geometry with respect to the indole plane. The result is shown in Fig. 10 (broken lines). In this case, $\mathrm{a} \mathrm{V}_{3}$ barrier of $1500 \mathrm{~cm}^{-1}$ is computed. Both potential energy curves (completely relaxed and methoxy group in the indole plane) have maxima at $-120^{\circ}, 0^{\circ}$, and $+120^{\circ}$, although the respective geometries are very different (see above). Obviously, the torsional axis changes its orientation during the torsional motion and the torsional constant $F$ is not conserved along this path. Therefore, the usual perturbative approaches for calculating torsional barriers do not apply and the above estimates for barriers and torsional constants give merely orders of magnitude.

A reduction of the $\mathrm{V}_{3}$ torsional barrier by coupling to other motions can therefore explain the observed splitting. Now, the exact nature of the coupling mode has to be determined. The out-of-plane motion, which gives a large contribution to the relaxed PES scan in Fig. 10, cannot be the sought-after mode since the respective torsional $\left(\mathrm{V}_{2}\right)$ mode is calculated at much lower energy $\left(Q_{54}\right.$ at $\left.77 \mathrm{~cm}^{-1}\right)$. From the normal mode analysis, it can be inferred that in the region of $500 \mathrm{~cm}^{-1}$ the only mode, which might be responsible for the coupling to the torsion, is the $\mathrm{C}-\mathrm{O}-\mathrm{C}$ in-plane bending of the methoxy group $\left(Q_{34}\right)$. We calculated the two-dimensional (2D) potential energy surface along the bending and the torsional coordinate at the MP2/6-311G(d,p) level of theory. Figure 11 shows the results obtained. The minima are found at a bending angle of $115^{\circ}$, while the saddle points are lo- 


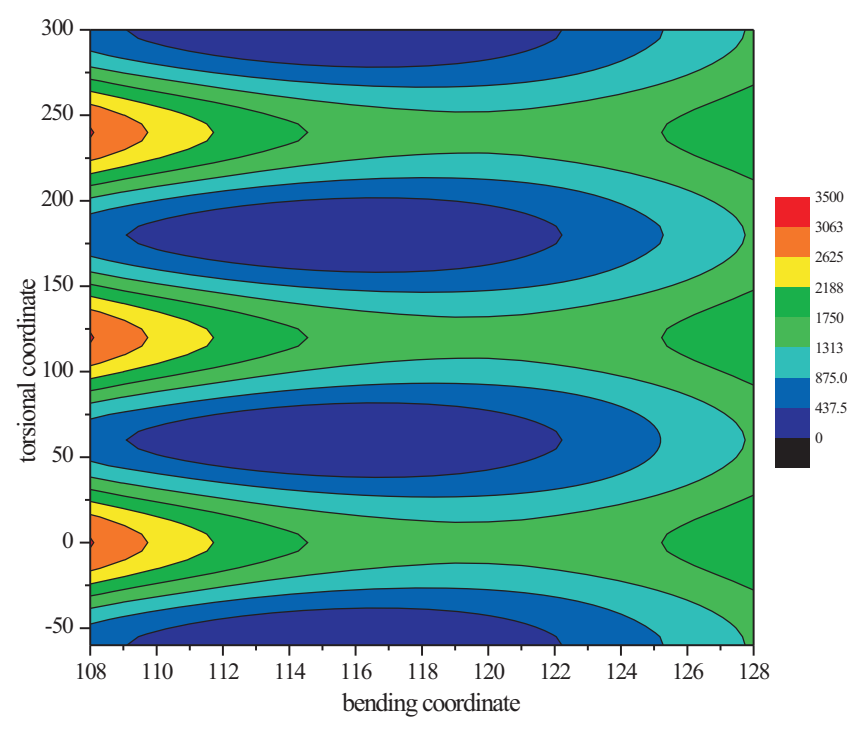

FIG. 11. MP2/6-311G(d,p) calculated two-dimensional potential energy surface along the methoxy in-plane bending and the methyl torsional coordinates. The values used for color coding are in $\mathrm{cm}^{-1}$.

cated at $120^{\circ}$. Thus, it seems very plausible that the splitting of the $502 \mathrm{~cm}^{-1}$ band is caused by the methyl torsional motion, whose barrier is lowered by the coupling to the methoxy in-plane bending mode. To test this hypothesis, we fit the spectrum of the $502 \mathrm{~cm}^{-1}$ band to a torsion-rotation Hamiltonian for a threefold torsion using a perturbative approach. $^{44,45}$ The goodness of this fit to an internal rotation model is even better than for a separate fit of the two components. Details of the fit are given in the online supporting material. $^{46}$

Another possibility for explaining the observed vibronic splitting might simply be a coincidence of two (different) vibronic bands, which are energetically very close. The (harmonic) state density calculated using the ${ }^{1} L_{b}$ frequencies gives a value of less than one state per wavenumber at an excess energy of $500 \mathrm{~cm}^{-1}$. The state density increases to about one state per wavenumber at $700 \mathrm{~cm}^{-1}$ and to more than five states per wavenumber above $1000 \mathrm{~cm}^{-1}$. So, it seems unlikely that a high state density is responsible for the observed splitting at $502 \mathrm{~cm}^{-1}$.

The $708 \mathrm{~cm}^{-1}$ band (Fig. 9) is split into two components, such as the $502 \mathrm{~cm}^{-1}$ band, with a splitting of 1026 $\mathrm{MHz}$. If the reason for the splitting is the torsional barrier of the methoxy group, a value of $\mathrm{V}_{3}$ of $290 \mathrm{~cm}^{-1}$ in the electronically excited state would result. On the other hand, we have a state density at $700 \mathrm{~cm}^{-1}$ which already makes it plausible that the splitting is due to a fortuitous coincidence of two vibronic bands. On the basis of the present experimental results, it is not possible to distinguish between these two possibilities. Nevertheless, a fit to the model Hamiltonian for internal rotation as for the $502 \mathrm{~cm}^{-1}$ band leads to a considerably poorer standard deviation, giving strong indication, that the reason for the appearance of a second band at $708 \mathrm{~cm}^{-1}$ is due to the state density here.

The changes of the rotational constants of the two components of the $899 \mathrm{~cm}^{-1}$ band are very different (cf. Table II). If the torsional barrier in the excited state is high, as can be assumed from the small splitting of $250 \mathrm{MHz}$, the rota- tional constants of the two subbands should be similar, since they are not strongly perturbed. The large difference in the rotational constants in both subbands makes it very improbable that the reason for the occurrence of two bands at $899 \mathrm{~cm}^{-1}$ is a torsional splitting, as proposed for the $502 \mathrm{~cm}^{-1}$ and possibly the $708 \mathrm{~cm}^{-1}$ bands.

\section{CONCLUSIONS}

Rotationally resolved electronic spectra of several vibronic bands in 5MOI have been obtained up to $1000 \mathrm{~cm}^{-1}$ in excess energy above the zero-point vibrational level of the first excited singlet state. From the measured values of the ground state rotational constants, it is shown that all observed bands belong to the anti-conformer of 5MOI. It is also shown that all observed bands have similarly oriented TDMs, consistent with the assignment of the first excited singlet state as the ${ }^{1} L_{b}$ state. In contrast, the parent molecule indole exhibits a TDM which strongly depends on the nature of the excited vibrational mode, owing to vibronic coupling with the ${ }^{1} L_{a}$ state. ${ }^{17,18}$

The reason for this different behavior is the large energy gap between the ${ }^{1} L_{b}$ and the ${ }^{1} L_{a}$ states of more than $4000 \mathrm{~cm}^{-1}$ in 5MOI, while this gap is only $1400 \mathrm{~cm}^{-1}$ in indole. Also the higher lying $B_{a}$ and $B_{b}$ states might contribute to the vibronic coupling owing to their larger oscillator strengths, which can compensate for their larger energy gap.

Within the numerical differentiation scheme we adopted for computation of Herzberg-Teller coupling, the CI wave functions are variationally optimized in a CSF basis for each distorted geometry. The change of the configuration state vector with nuclear geometry may, in principle, be caused by each of the states, which are present in the basis. Breaking down the Herzberg-Teller activity into contributions from individual excited states would require an expansion of the Herzberg-Teller derivatives in sum over states, what is beyond the scope of this study.

A splitting in some of the vibronic bands in the spectrum of 5MOI is analyzed in terms of a torsional splitting due to the threefold rotation of the methoxy group. Although the barrier for this motion is high, both in the ground and the excited states, a lower effective barrier in the excited state is postulated to be caused by excitation of a vibration which is strongly coupled to the torsion. According to our analysis, the $502 \mathrm{~cm}^{-1}$ band is due to vibration $Q_{34}$, which is the $\mathrm{C}-\mathrm{O}-\mathrm{C}$ bending vibration of the methoxy group. It seems plausible that this vibration couples strongly to the torsional motion, giving rise to a saddle point on the two-dimensional potential energy surface, which lies lower than the transition state on the torsional potential energy curve. The 2D surface along the respective modes in the ground state shows exactly the assumed behavior. The excited state lifetimes of the vibronic bands of 5MOI have been determined to be between 6 and $10 \mathrm{~ns}$, with the exception of the $700 \mathrm{~cm}^{-1}$ band, which shows a sharp drop to only $2.4 \mathrm{~ns}$. Thus, this mode seems to be an effective promoter mode for internal conversion to the electronic ground state of 5MOI. A calculation of the DFT/ MRCI energies of the $S_{0}$ and $S_{1}$ potential energy surfaces 
along mode $Q_{32}$ shows a curve crossing of the two PES. Further work to elucidate this question is underway.

\section{ACKNOWLEDGMENTS}

This work was supported by the U.S. National Science Foundation (Contract No. CHE-0911117), the Netherlands Organization for Scientific Research (NWO), and the Deutsche Forschungsgemeinschaft in the framework of the NWO-DFG bilateral program [Grant No. SCHM1043/10 (Germany) and Grant No. DN 72-248 (The Netherlands)]. The authors would like to thank the National Computer Facilities of the Netherlands Organization of Scientific Research (NWO) for a grant on the Dutch supercomputing facility SARA. Granted computing time at Universitätsrechenzentrum Köln is gratefully acknowledged.

${ }^{1}$ D. M. Sammeth, S. Yan, L. H. Spangler, and P. R. Callis, J. Phys. Chem. 94, 7340 (1990).

${ }^{2}$ M. R. Eftink, L. A. Selvidge, P. R. Callis, and A. A. Rehms, J. Phys. Chem. 94, 3469 (1990).

${ }^{3}$ B. Albinsson and B. Nordén, J. Phys. Chem. 96, 6204 (1992).

${ }^{4}$ T. L. O. Barstis, L. I. Grace, T. M. Dunn, and D. L. Lubman, J. Phys. Chem. 97, 5820 (1993).

${ }^{5}$ B. J. Fender, D. M. Sammeth, and P. R. Callis, Chem. Phys. Lett. 239, 31 (1995).

${ }^{6}$ G. Berden, W. L. Meerts, and E. Jalviste, J. Chem. Phys. 103, 9596 (1995).

${ }^{7}$ C. Kang, T. M. Korter, and D. W. Pratt, J. Chem. Phys. 122, 174301 (2005).

${ }^{8}$ P. R. Callis, J. T. Vivian, and L. S. Slater, Chem. Phys. Lett. 244, 53 (1995).

${ }^{9}$ A. C. Borin and L. Serrano-Andrés, Chem. Phys. 262, 253 (2000).

${ }^{10}$ L. Serrano-Andrés and A. C. Borin, Chem. Phys. 262, 267 (2000).

${ }^{11}$ A. L. Sobolewski and W. Domcke, Chem. Phys. Lett. 315, 293 (1999).

${ }^{12}$ L. Serrano-Andrés and B. O. Roos, J. Am. Chem. Soc. 118, 185 (1996)

${ }^{13}$ M. R. Eftink, Methods Biochem. Anal 35, 127 (1991).

${ }^{14}$ J. Lakowicz, Principles of Fluorescence Spectroscopy, 2nd ed. (Plenum, New York, 1999).

${ }^{15}$ J. R. Platt, J. Chem. Phys. 17, 484 (1949).

${ }^{16}$ G. Weber, Biochem. J. 75, 335 (1960).

${ }^{17}$ C. Brand, J. Küpper, D. W. Pratt, W. L. Meerts, D. Krügler, J. Tatchen, and M. Schmitt, Phys. Chem. Chem. Phys. 12, 4968 (2010).

${ }^{18}$ J. Küpper, D. W. Pratt, W. L. Meerts, C. Brand, J. Tatchen, and M. Schmitt, Phys. Chem. Chem. Phys. 12, 4980 (2010).

${ }^{19}$ M. Martinaud and A. Kadiri, Chem. Phys. 28, 473 (1978).
${ }^{20}$ Y. Yamamoto and J. Tanaka, Bull. Chem. Soc. Jpn. 45, 1362 (1972).

${ }^{21}$ J. W. Hager, D. R. Demmer, and S. C. Wallace, J. Phys. Chem. 88, 5513 (1984).

${ }^{22}$ Y. Huang and M. Sulkes, J. Phys. Chem. 100, 16479 (1996).

${ }^{23}$ G. M. Florio, R. A. Christie, K. D. Jordan, and T. S. Zwier, J. Am. Chem. Soc. 124, 10236 (2002).

${ }^{24}$ S. Wiedemann, A. Metsala, D. Nolting, and R. Weinkauf, Phys. Chem. Chem. Phys. 6, 2641 (2004).

${ }^{25}$ M. Schmitt, J. Küpper, D. Spangenberg, and A. Westphal, Chem. Phys. 254, 349 (2000).

${ }^{26} \mathrm{~S}$. Gerstenkorn and P. Luc, Atlas du spectre d'absorption de la molécule d'iode (CNRS, Paris, 1982).

${ }^{27}$ R. Ahlrichs, M. Bär, M. Häser, H. Horn, and C. Kölmel, Chem. Phys. Lett. 162, 165 (1989).

${ }^{28}$ A. Schäfer, C. Huber, and R. Ahlrichs, J. Chem. Phys. 100, 5829 (1994).

${ }^{29}$ C. Hättig and F. Weigend, J. Chem. Phys. 113, 5154 (2000).

${ }^{30}$ C. Hättig and A. Köhn, J. Chem. Phys. 117, 6939 (2002).

${ }^{31}$ C. Hättig, J. Chem. Phys. 118, 7751 (2003).

${ }^{32}$ S. Grimme and M. Waletzke, J. Chem. Phys. 111, 5645 (1999).

${ }^{33}$ A. D. Becke, J. Chem. Phys. 98, 1372 (1993).

${ }^{34}$ C. Lee, W. Yang, and R. Parr, Phys. Rev. B 37, 785 (1988).

${ }^{35}$ A. Ostermeier, A. Gawelczyk, and N. Hansen, Step-Size Adaptation Based on Non-Local Use of Selection Information, Lecture Notes in Computer Science: Parallel Problem Solving from Nature (PPSN III) (Springer, New York, 1994), pp. 189-198.

${ }^{36}$ N. Hansen and A. Ostermeier, Evol. Comput. 9, 159 (2001).

${ }^{37}$ A. Suwaiyan and M. A. Morsy, Spectrochim. Acta, Part A 53, 575 (1997).

${ }^{38}$ G. Herzberg, Molecular Spectra and Molecular Structure, III. Electronic Spectra and Electronic Structure of Polyatomic Molecules (Van Nostrand Reinhold, New York, 1966).

${ }^{39}$ M. Böhm, J. Tatchen, D. Krügler, K. Kleinermanns, M. G. D. Nix, T. A. LeGreve, T. S. Zwier, and M. Schmitt, J. Phys. Chem. A 113, 2456 (2009).

${ }^{40}$ J. T. Hougen and J. K. G. Watson, Can. J. Phys. 43, 298 (1965).

${ }^{41}$ A. Held, B. B. Champagne, and D. W. Pratt, J. Chem. Phys. 95, 8732 (1991).

${ }^{42}$ R. Pariser, J. Chem. Phys. 24, 250 (1956).

${ }^{43}$ H. Tylli and H. Konschin, J. Mol. Spectrosc. 42, 7 (1977).

${ }^{44}$ W. Gordy and R. L. Cook, Microwave Molecular Spectra, 3rd ed. (Wiley, New York, 1984).

${ }^{45}$ D. R. Herschbach, J. Chem. Phys. 31, 91 (1959).

${ }^{46}$ See supplementary material at http://dx.doi.org/10.1063/1.3459128 for the geometry parameters of the three lowest singlet states of the syn and anti conformers of 5MOI from the CC2/cc-pVTZ optimized structures, details on the fit of the split band at $502 \mathrm{~cm}^{-1}$ to a torsional Hamiltonian, and the rovibronic spectra of the vibronic bands at 899 and $1016 \mathrm{~cm}^{-1}$ above the electronic origin at $33120.53 \mathrm{~cm}^{-1}$. 